\title{
Mira's wind explored in scattering infrared CO lines
}

\author{
N. Ryde, B. Gustafsson and K. Eriksson \\ Uppsala Astronomical Observatory, Box 515, S-751 20, Uppsala, Sweden \\ ryde, bg, and ke@astro.uu.se \\ and \\ K. H. Hinkle \\ National Optical Astronomy Observatories ${ }^{1}$, P.O. Box 26732, Tucson, AZ 85726, U.S.A. \\ hinkle@noao.edu
}

\begin{abstract}
We have observed the intermediate regions of the circumstellar envelope of Mira (o Ceti) in photospheric light scattered by three vibration-rotation transitions of the fundamental band of $\mathrm{CO}$, from low-excited rotational levels of the ground vibrational state, at an angular distance of $\beta \sim 2^{\prime \prime}-7^{\prime \prime}$ away from the star. The data were obtained with the Phoenix spectrometer mounted on the $4 \mathrm{~m}$ Mayall telescope at Kitt Peak. The spatial resolution is approximately $0.5^{\prime \prime}$ and seeing limited. Our observations provide absolute fluxes, leading to an independent new estimate of the mass-loss rate of approximately $3 \times 10^{-7} \mathrm{M}_{\odot} \mathrm{yr}^{-1}$, as derived from a simple analytic wind model. We find that the scattered intensity from the wind of Mira for $2^{\prime \prime} \lesssim \beta \lesssim 7^{\prime \prime}$ decreases as $\beta^{-3}$, which suggests a time constant mass-loss rate, when averaged over 100 years, over the past 1200 years.
\end{abstract}

Subject headings: stars: AGB and post-AGB — circumstellar matter — stars: individual (o Ceti) stars: late-type — stars: mass loss — infrared: stars

\section{INTRODUCTION}

Ever since the circumstellar lines of the $\alpha$ Her $M$ supergiant were identified in absorption against the photospheric continuum of the G III secondary (Deutsch 1956), there have been numerous attempts to map circumstellar regions. Three spectroscopic tools have been used extensively. The most extensively employed are microwave emission lines. The IR continuum from circumstellar dust, especially in the observations by the IRAS and ISO satellites, has been used. Finally, approximately a dozen circumstellar shells have been imaged in photospheric light scattered by atomic resonance lines. In this paper we shall extend the

\footnotetext{
${ }^{1}$ Operated by the Association of Universities for Research in Astronomy, Inc. under cooperative agreement with the National Science Foundation
}

latter technique to resonance scattering in molecular lines using the mid-infrared vibration-rotation lines of the ubiquitous $\mathrm{CO}$ molecule.

In his review of circumstellar envelopes and AGB stars, Habing (1996) noted both the usefulness and the uniqueness of measuring scattered resonance lines, for example of NaI and KI, but drew attention to the relatively few cases where this method has been applied. This is certainly because the observations and their interpretation are quite complex. There are uncertainties in relating these observations to the mass and structure of the expanding gas since atoms are depleted by dust grains and the details of ionization in the circumstellar shell are not known. An advantage of microwave measurements is that the molecular species sampled may be much less affected by these uncertainties. This advantage is also present in the 
study of photospheric light scattered by circumstellar CO molecules, as presented in this paper. The spectral energy distributions (SEDs) of AGB stars have a maximum in the infrared which also suggests infrared CO observations. The infrared vibration-rotation lines of $\mathrm{CO}$, as an alternative to rotational $\mathrm{CO}$ lines at millimeter wavelengths, also allow the study of regions close to the star and admit higher spatial resolution in single telescope studies.

Pioneering investigations of atomic resonance line scattering were made of the KI $7699 \AA$ circumstellar emission in $\alpha$ Ori by Bernat \& Lambert $(1975,1976)$. Recent work includes the study of circumstellar shells around three N-type carbon stars using Ki $7699 \AA$ (Gustafsson et al. 1997) and of circumstellar shells of M-type mira stars using various atomic resonance lines (Plez \& Lambert 1994; Guilain \& Mauron 1996).

Imaging off-star emission of $\mathrm{CO}$ at $4.6 \mu \mathrm{m}$ has previously only been done a few times (Sahai \& Wannier 1985; Ryde et al. 1999). Dyck et al. (1983) performed speckle interferometry on a number of CO fundamental vibrational-rotational lines. Sahai \& Wannier studied the intermediate regions of the circumstellar envelope of the dust-enshrouded and bright IR star CW Leonis (IRC+10216) by using an annular aperture, and determined a kinetic temperature of its shell at an average radius of $2^{\prime \prime}$. This technique provided only spatially averaged fluxes but led the authors also to the conclusion that the mass-loss rate in the inner parts of the circumstellar shell is less than that corresponding to the region observed in millimeter CO lines. Since the Sahai and Wannier observations, great advances have been made in infrared detectors. It is, however, not trivial to apply infrared arrays in high resolution spectroscopy since at wavelengths longer than about $1.6 \mu \mathrm{m}$ thermal radiation from room temperature spectrometers dominates the stellar signals. Cryogenic spectrometers are therefore required, such as the Phoenix spectrometer (Hinkle et al. 1998). One of the justifications for building this spectrometer was, in fact, to map circumstellar shells as presented in this paper. In Ryde et al. (1999) we demonstrated methods of observation and analysis of the vibration-rotation $\mathrm{CO}$ emission lines from the intermediate regions of circumstellar winds. Here, we present results of an application of these methods to the circumstellar envelope of the M4-7IIIe giant o Ceti (Mira).

Section 2 discusses Mira. Sections 3 and 4 describe the observational set-up and the reduction procedures, and the absolute flux calibration, respectively. Section 5 reviews the observational results and Section 6 discusses these results in the light of an analytic model for circumstellar envelopes.

\section{MIRA}

A study of the circumstellar envelope of $o$ Ceti (Mira or HD 14386) is of interest for several reasons. The star is the prototype of the mira-class of long-period variables characterized by large amplitude visual variations. In the case of $o$ Ceti the $\mathrm{V}$-band amplitude is more than 6 magnitudes and the mean period is 332 days. The miras are cool AGB stars with most of the energy emitted in the infrared. The brightness of Mira reflects its closeness, $(128 \pm 18)$ pc (ESA 1997). The stellar mass loss produces a circumstellar envelope which, at the distance of Mira, has an angular extension of $2^{\prime}$ on the sky (Loup et al. 1993). Note, however that $o$ Ceti departs from many miras in being a binary system. The angular distance between Mira A and the hot, compact companion star Mira B (VZ Ceti) is 0.6" (Karovska et al. 1993; 1997). The companion could be a white dwarf with a mass of about $1 \mathrm{M}_{\odot}$, a luminosity of $2 \mathrm{~L}_{\odot}$, and a temperature of more than $30000 \mathrm{~K}$, which is embedded in an accretion disk, giving rise to an abnormal illumination of Mira A (Danchi et al. 1994). Also, note that a number of other high-resolution, optical and infrared wavelength measurements provide evidence that Mira itself is elongated (see discussion by Ryde \& Schöier (2000) and references therein).

Theoretical model calculations (Bowen 1988; Höfner \& Dorfi 1997; Höfner et al. 1998; see Woitke (1998) for a review) indicate that the mass loss of these stars is most probably caused by a combination of radial pulsation and radiation pressure on dust grains and/or molecules. It may seem natural to expect this mass loss to be spherically symmetric, but stellar rotation may introduce a latitude dependence (Dorfi \& Höfner 1996). Also, on the scale of the spatial resolution of our observations, corresponding to a time-scale of 
about 100 years, one could expect the wind to be homogeneous (observations of Mira show the current pulsational behavior dates back to at least 1638).

However, there are indications from radio observations that the wind of Mira departs from this simple picture. There is an asymmetry and a shoulder clearly visible in the radio-line profiles, see for example Planesas et al. (1990a). There have been several suggestions in the literature of different multi-wind scenarios or other phenomena introducing dramatic variations in the envelope structure with distance in order to fit the profiles. There exist several combinations of massloss rate, expansion and turbulent velocities that are able to reproduce the Mira circumstellar line profiles rather well and as a result there is a lack of consensus regarding the actual expansion velocities of Mira's wind. All these results are based on microwave and sub-millimeter observations of rotational CO lines. Crosas et al. (1997) experiment with $\mathrm{v}_{\mathrm{exp}} \approx 2$ and $\mathrm{v}_{\text {turb }} \approx 4 \mathrm{~km} \mathrm{~s}^{-1}$ for their inner wind, while Young (1995) arrives at $\mathrm{v}_{\exp }=4.8 \mathrm{kms}^{-1}$ from a fit. However, he points out that the wings show an expansion velocity of $10 \mathrm{kms}^{-1}$. Knapp et al. (1998) model the circumstellar envelope (CSE) with a fast outer wind with a mass-loss rate resembling single wind miras, $\dot{\mathrm{M}}=4.4 \cdot 10^{-7} \mathrm{M}_{\odot} \mathrm{yr}^{-1}$, and an expansion velocity of $(6.7 \pm 1.0) \mathrm{km} \mathrm{s}^{-1}$. The inner wind is supposed to be a resumed wind in analogy with the detached shells found in four carbon stars (Olofsson et al. 1996). This slow wind component has a lower mass-loss rate and a much lower expansion velocity than what is found normally in miras; $\dot{\mathrm{M}}=$ $9.4 \cdot 10^{-8} \mathrm{M}_{\odot} \mathrm{yr}^{-1}$ and $\mathrm{v}_{\exp }=(2.4 \pm 0.4) \mathrm{km} \mathrm{s}^{-1}$. Planesas et al. (1990a) interpret their observations of $\operatorname{CO}(J=1-0)$ and $\operatorname{CO}(J=2-1)$ lines as partly originating from a dominating, spherically symmetric CSE with $\mathrm{v}_{\exp }=3 \mathrm{~km} \mathrm{~s}^{-1}$ and a partial collimation of the stellar wind into an additional bipolar lobe.

Obviously it would be of great interest to study whether the peculiarities observed in the microwave lines are also reflected in the infrared $\mathrm{CO}$ vibration-rotation lines, which map the intermediate regions (approximately $100-1000$ stellar radii) of Mira's circumstellar environment.

The geocentric, radial velocity of Mira is large enough for the stellar CO lines to be shifted out of the telluric lines. This is a key factor since the blending of the telluric and stellar lines makes the analysis of the faint circumstellar emission very difficult.

Multi-wavelength angular diameter measurements of Mira (Haniff et al. 1995) combined with Hipparcos trigonometric parallaxes (Van Leeuwen et al. 1997) suggest a mean radius of $(464 \pm 80) R_{\odot}$. Based on the work of Mahler et al. (1997) on radius and luminosity variations of Mira from Wing near-IR photometry, one can derive a bolometric luminosity $\mathrm{L}_{\text {tot }}=8900 \mathrm{~L}_{\odot}$, a temperature $\mathrm{T}_{\text {eff }}=$ $2400 \mathrm{~K}$, and a radius of $550 \mathrm{R}_{\odot}=3.8 \cdot 10^{13} \mathrm{~cm}$ for the phase of $o$ Ceti on the date our observations were made, 1998 October 28-29. These values are consistent with the corresponding values of, for example, Danchi et al. (1994) and Haniff et al. (1995). They arrive at similar temperatures; a mean (over phases) effective temperature of about $\mathrm{T}_{\text {eff }} \approx 2800 \mathrm{~K}$. We note, however, that this value is low as compared with the values suggested by Perrin et al. (1998) for giants in the interval M4IIIM7III which is characteristic of miras. Assuming a mass of $\mathcal{M} \approx 1.0 \mathrm{M}_{\odot}$, we find the logarithmic surface gravity, $\log g=\log \left(\mathrm{GM} / \mathrm{R}_{*}^{2}\right)$, to be approximately $-1.0[\mathrm{cgs}]$.

\section{OBSERVATIONAL SET-UP AND RE- DUCTIONS}

The observations were carried out in 1998 on October 28 and 29 using the 4 meter Mayall telescope at Kitt Peak with the Phoenix spectrometer, mounted at the Cassegrain focus of the equatorially mounted telescope. This cryogenic, singleorder echelle spectrometer is a long-slit, high spectral resolution instrument, designed for the $1-$ $5 \mu \mathrm{m}$ region (Hinkle et al. 1998) and marks a major achievement. At $4.6 \mu \mathrm{m}$ it is now possible to observe objects 5 magnitudes fainter than was possible with the Fourier Transform Spectrometer on the same telescope.

The detector is an Aladdin $512 \times 1024$ element InSb array cooled to $35 \mathrm{~K}$. The rest of the spectrometer is cooled to $50 \mathrm{~K}$. For our observations, a $30^{\prime \prime}$ long and $0.4^{\prime \prime}$ (2 pixels) wide entrance slit was used, resulting in a spectral resolution of around $\mathrm{R}=60000$. The slit width projection onto the sky of $0.4^{\prime \prime}$ means that the spectral information is an average over, at least, a region of this size. 
The visual seeing was around $0.8^{\prime \prime}$, but we note that the seeing disk in the infrared $(4.6 \mu \mathrm{m})$ is $60 \%$ of that in the visual (Léna 1988), so that the seeing nearly matched the slit size, which is approximately $30 \%$ greater than the diffraction limit of the telescope. The dispersion of the spectrograph leads to an over-sampled observed spectrum and each spectrum covers a very small wavelength range; approximately $11 \mathrm{~cm}^{-1}$.

We observed the circumstellar envelope of $o$ Ceti in a hashed configuration, resembling a perpendicular railway crossing with the star in the middle, see Figure 1. The long slit was placed in the off-star position at $2^{\prime \prime}$ away from the star. We were able to detect emission to a position corresponding to a maximum distance from the star of $7^{\prime \prime}$. The four positions will overlap at a distance of approximately $\sqrt{2^{2}+2^{2}}$ " away from the star, which enables us to make a relative calibration of the observations, for the different slit positions around the star.

We chose an echelle setting for observing low excitation lines of the vibration-rotation, fundamental R-branch of the electronic ground state of ${ }^{12} \mathrm{C}^{16} \mathrm{O}$. The lines selected, 1-0 $\mathrm{R}(1)$ (2150.86 $\mathrm{cm}^{-1}$, i.e. $\mathrm{v}=1 \rightarrow 0$ and $J^{\prime}=2 \rightarrow J^{\prime \prime}=1$ ), 1-0 R(2) (2154.60 $\mathrm{cm}^{-1}$ ), and 1-0 R(3) (2158.30 $\mathrm{cm}^{-1}$ ), have minimal interference with telluric lines. The potentially important $1-0 \mathrm{R}(0)$ line has an interfering telluric water line. Thus, we observed the region 2150 to $2160 \mathrm{~cm}^{-1}$, the spectral coverage being limited by the array length. The spectrometer works in order 12 at these wavelengths.

The CO fundamental bands are located in the thermal infrared where the sky background radiation is high and somewhat variable. Phoenix is not a sky balanced device, which means that the thermal radiation is recorded. At the $4.6 \mu \mathrm{m}$ wavelength of $\mathrm{CO}$, the thermal background radiation at Kitt Peak would limit exposure times at $\mathrm{R}=60000$ to about ten minutes. However, the $4.6 \mu \mathrm{m}$ spectrum has a contribution of telluric lines, mostly $\mathrm{CO}$ and $\mathrm{H}_{2} \mathrm{O}$, which are seen in emission. To avoid saturation of the telluric lines the exposure time was limited to 60 seconds.

Our observations consist of two types: on-star and off-star exposures. On-star exposures are required to remove scattered light from the off-star exposures. In order to remove the thermal spec- trum from the source spectrum, two exposures are required, one of the source and one of the sky (Joyce 1992). All spectra must also have array pixel sensitivity differences removed using flats and darks as described by Joyce (1992). Since Phoenix has a long slit, nodding along the slit is possible for point sources. Therefore, on-star spectra were taken by moving the star along the slit by $15^{\prime \prime}$ between exposures. The brightness of Mira limited these exposures to $30 \mathrm{~s}$. Each set of exposures then gave, after differencing, two background-subtracted, on-star spectra. Since the stellar signal is extincted by the optical depth of the telluric lines, the background-subtracted spectra show in absorption the telluric lines which appear in emission in the raw images.

Off-star frames were taken the first night by alternatively observing $2^{\prime \prime}$ south and making an identical background observation $62^{\prime \prime}$ south of the star, both with an exposure time of $60 \mathrm{~s}$. o Ceti has a CO shell with a radius of $57^{\prime \prime}$, as measured by radio observations (Loup et al. 1993). The total effective observing time in the south position was 66 minutes. Corresponding observations were subsequently made in the north position, $2^{\prime \prime} \mathrm{N}$, also here with an exposure time per nod of $60 \mathrm{~s}$. The total effective observing time in the north position was 80 minutes. The second night a more efficient nodding algorithm was employed. Off-star frames were taken by alternatively observing $2^{\prime \prime}$ east and $2^{\prime \prime}$ west, $60 \mathrm{~s}$ each, then a background observation was taken $60^{\prime \prime}$ west off the star. A total of 54 effective minutes were observed in both the west and east positions. Over the few minute (at maximum) interval between the source and sky observations the sky background level is almost constant and the airmass changes only slightly. While the thermal radiation and extinction of well-mixed telluric gases, such as CO, are scaled with airmass and time, water is not, on most nights being in the form of clouds visible in the infrared. As a result the telluric water vapor lines will not cancel cleanly in the subsequent differencing of spectra.

The on-star spectra were used to remove the effects of scattered stellar light in the terrestrial atmosphere and in the spectrometer. Thus, these were normalized to unit level and then scaled to the levels of the off-star spectra. The circumstellar $\mathrm{CO}$ vibration-rotation emission was obtained by subtracting the scaled on-star spectra from the off- 
star ones. The emission we wish to detect is about 50 times weaker than the on-star intensity. The on-star intensity is typically about twenty to thirty times brighter than the sky background.

The reduction of the data was performed using standard routines of the latest version of IRAF (Image Reduction and Analysis Facility). The wavelength calibration was made by using the telluric lines in every frame. The accuracy of the wavelength calibration is of the same order as the resolution. Therefore, the uncertainty in the wavelength scale is probably approximately $0.04 \mathrm{~cm}^{-1}$ or $6 \mathrm{~km} \mathrm{~s}^{-1}$.

From the ISO data archive $^{2}$ we have retrieved the spectrum around and the flux at $4.6 \mu \mathrm{m}$ of Mira, obtained with the Infrared Space Observatory (Kessler et al. 1996). The reductions were made using the most recent pipeline basic reduction package OLP (v.7) and the ISO Spectral Analysis Package (ISAP v.1.5). The pipeline processing of the data, such as the flux calibration, is described by de Graauw et al. (2000); the combined absolute and systematic uncertainties in the fluxes are of the order of $\pm 10 \%$.

\section{ABSOLUTE FLUX CALIBRATION}

The absolute calibration of the spectra in the $4 \mu \mathrm{m}$ region was made by a comparison of the measured on-star flux (in counts) with the absolute flux (in physical units) expected in the broad Mband. The conversion factors obtained are then used for calibrating the off-star spectra. This method contains a number of uncertain steps, related to the variability of Mira, the uncertainties in the calibration of broad-band photometry, the scaling from the broad-band to the narrow wavelength region observed spectroscopically, the question of how much stellar light was caught within the spectrograph slit in the on-star observation, and the question whether the sensitivity of the detector or the transmission of the Earth's atmosphere might have changed between the on-star and off-star observations. In order to check the calibration we therefore compared with ISO fluxes and made a final test in a comparison between the

${ }^{2}$ Partly based on observations with ISO, an ESA project with instruments funded by ESA Member States and with the participation of ISAS and NASA. http://isowww.estec.esa.nl/ observed spectrum and a calculated one from a model atmosphere, allowing for the stellar radius and the distance as measured by the Hipparcos satellite.

The amplitude of the Mira light curve is visually large, but decreases rapidly towards longer wavelengths. From the NASA Catalog of Infrared Observations (Gezari et al. 1984) a mean of eleven measurements gives a flux in the Johnson $\mathrm{M}$ filter of $\log \mathcal{F}_{\nu}=3.6 \pm 0.2$ [Jy]. This M-band mean flux is easily converted to $\left(5.6_{-2.1}^{+3.3}\right) \times 10^{-7} \mathrm{erg} \mathrm{s}^{-1} \mathrm{~cm}^{-2} \mu \mathrm{m}^{-1}$ or, since 1 pixel corresponds to $0.26 \AA,\left(1.5_{-0.5}^{+0.8}\right) \times$ $10^{-11} \mathrm{erg} \mathrm{s}^{-1} \mathrm{~cm}^{-2}$ pixel $^{-1}$. In view of the fact that the flux is peaked towards the blue end of the M-band in such a way that the flux in the spectroscopic band is higher than the mean flux in the band (cf. Figure 2), one must correct the absolute calibration. From the lowresolution ISO spectrum of this region of Mira we find a correction factor of 1.23. We obtained this correction by computing a mean of the ISO spectrum over the M-band and comparing with the ISO flux at $4.64 \mu \mathrm{m}$. Our model atmosphere (see discussion below) gives a similar result. Thus, for October 28 we arrive at a conversion factor of $\left(3.3_{-1.2}^{+1.9}\right) \times 10^{-14} \mathrm{erg} \mathrm{cm}^{-2}$ per detector count and for October 29 we get a factor of $\left(2.7_{-1.0}^{+1.6}\right) \times 10^{-14} \mathrm{erg} \mathrm{cm}^{-2}$ per detector count. This leads to a flux in the $4.64 \mu \mathrm{m}$ band of $4900 \mathrm{Jy}$ or $6.9 \times 10^{-7} \mathrm{erg} \mathrm{s}^{-1} \mathrm{~cm}^{-2} \mu \mathrm{m}^{-1}$. This may readily be compared with the ISO flux at the same wavelength, $(4700 \pm 400) \mathrm{Jy}$, cf. Section 3 .

In order to check the calibration further we also used an OS-MARCS spherical model-atmosphere for the stellar parameters $\mathrm{T}_{\text {eff }}=2400 \mathrm{~K}, \log g=-1.0$ [cgs], and solar metallicity and allowing for the stellar radius $\left(\mathrm{R}_{*}=3.8 \cdot 10^{13} \mathrm{~cm}\right)$ and a Hipparcos distance $\mathrm{d}=128 \mathrm{pc}$, to generate a synthetic spectrum of the region of interest. The stellar parameters are determined for the phase of $o$ Ceti at October 29, 1998, the date the NIR observations were made, see Ryde \& Schöier (2000). The model atmosphere is a part of a new grid of model atmospheres (Plez et al. 2000) being generated by an extensive update of the MARCS code and its input data (based on Gustafsson et al. 1975). Molecular lines of $\mathrm{H}_{2} \mathrm{O}$ (with line lists of Partridge and Schwencke 1997), of CO (lists of Goorvitch 1994), and of many other molecules, are taken into ac- 
count in the spherical radiative transfer for the calculation of a synthetic spectrum.

Figure 3 shows the spectrum generated from the model atmosphere and a comparison with the observed one. The general features are well reproduced and are mainly due to photospheric water vapor and CO. The agreement of the over-all flux level is astonishingly good; the model flux is around $30 \%$ too small. We find this agreement between the empirical, calibrated, absolute flux and the flux we calculate from a model atmosphere very satisfactory, in view of all the uncertainties anticipated.

It is worth noting that synthetic spectra from the new generation of spherical MARCS models in the near infrared are able to reproduce observed spectra fairly well, even for a pulsating star like Mira. While the cyclic variations in mira spectra are well known and conspicuous at high resolution (Hinkle et al. 1982; Hinkle et al. 1984), little is know about phase dependent variations of the 4.6 $\mu \mathrm{m}$ photospheric spectra of mira stars. It is possible that depths of formation of the continuum and lines at $4.6 \mu \mathrm{m}$ are in a region of the photosphere similar to that of the visual spectrum where the dynamic behavior does not have large effects on absorption line formation. However, it is also possible that the phase of observation gave a fortuitous match to the model. In either case the stellar atmosphere is extended and the MARCS match to the spectrum is impressive.

Figure 4 shows a blow-up of the photospheric CO $\mathrm{R}(2)$ absorption line as well as the scattered circumstellar $\mathrm{R}(2)$ emission. This emission line is shifted by $0.42 \mathrm{~cm}^{-1}$ compared to the telluric wavelength scale, corresponding to a radial velocity of $58.6 \mathrm{kms}^{-1}$. Mira has a $\mathrm{v}_{\mathrm{LSR}}=47 \mathrm{~km} \mathrm{~s}^{-1}$ deduced from radio data (Loup et al. 1993), which equals a heliocentric velocity of $57 \mathrm{~km} \mathrm{~s}^{-1}$. At the time of the observations and at Kitt Peak, this corresponds to a geocentric radial velocity of $59 \mathrm{kms}^{-1}$, in excellent agreement with the observations. Thus, the wavelength of the scattered light is centered on the laboratory wavelength corrected for the stellar radial velocity. The photospheric absorption lines, however, are also shifted due to the velocity of the pulsating photosphere. The bisector of the photospheric absorption line lies less than $2 \mathrm{kms}^{-1}$ blue-wards of the center of the emission line. The weak emission in the absorption line in the on-star spectrum is due to scattered light from the CSE. This light is included in the on-star measurement since the long-slit will also cover the CSE in two directions away from the star. Also present in the on-star spectrum is additional absorption along the line-of-sight through the circumstellar shell. In our measured, off-star, scattered CO light we do not correct for these components. However, they are of nearly equal intensity and should cancel at the level of the uncertainties of our measurements.

Note that when discussing relative fluxes, such as in Section 5 where the $\mathrm{R}(1) / \mathrm{R}(3)$ and $\mathrm{R}(2) / \mathrm{R}(3)$ ratios are calculated, the details in the flat-fielding may introduce uncertainties. The three lines span over the entire range of the detector, and therefore variable effects not taken care of by the flat-fielding will show up as an error in the ratios.

\section{OBSERVATIONAL RESULTS}

Figure 5 shows the off-star spectrum of the west position. Superimposed is the corresponding, scaled, on-star spectrum, which at least approximately represents the radiation exciting the molecules. The intensity of this spectrum is scaled in order to fit the general features in the offstar spectrum. The off-star spectrum obviously consists partly of stellar light that is scattered in the Earth's atmosphere, in the telescope, the spectrometer, and/or by dust grains in the circumstellar shell, leading to a spectrum resembling the on-star one. Nearly all features are identified as photospheric $\mathrm{CO}$ and $\mathrm{H}_{2} \mathrm{O}$ lines, cf. Section 4 and Figure 3. The on-star spectrum includes tens of $\mathrm{CO}$ vibration-rotation lines of various excited vibrational states. The cold off-star spectrum, however, includes only the 1-0 $\mathrm{R}(1)$, $\mathrm{R}(2)$, and $\mathrm{R}(3)$ vibration-rotation emission lines of ${ }^{12} \mathrm{CO}$ within the observed spectral range, except for the scattered, on-star light. Thus, the circumstellar molecules are radiatively excited by the stellar light, which is re-emitted as emission.

Figure 6 shows the resulting $\mathrm{CO}$ emission from the observations west, east, north and south of $o$ Ceti, integrated over the long slit. This emission is recovered from the data by subtracting the scaled on-star spectrum from the off-star one. The resulting emission lines are the circumstellar $\mathrm{R}(1)$, 
$\mathrm{R}(2)$ and $\mathrm{R}(3)$ lines. Variations in the telluric lines during the time between the object frame exposure and the background exposure and/or during the time between the on-star and off-star exposures would result in non-zero residuals. From the amplitudes of the signals of the CO emission lines, measured at different directions from the star, and the noise level as shown in Figure 7, we estimate a signal-to-noise ratio of approximately $5-15$. The noise arises partly from spurious mismatches in intensity between the on- and off-star spectra.

To study the emission as a function of the angular distance from the star, we divided our long-slit spectra into 79 sub-spectra (symmetrically around the maximum intensity representing the closest point to the star), one spectrum per pixel in the spatial direction. In this way we obtained 79 spectra for every off-star slit position, each corresponding to $0.2^{\prime \prime}$ on the sky, if seeing is neglected. For every spectrum, the intensity of the three CO vibration-rotation emission lines $[R(1), R(2)$ and $\mathrm{R}(3)$ ] from the wind are measured. These spectra provide data representing a distance range from $2^{\prime \prime}$ to a maximum of $7^{\prime \prime}$ away from the star, every slit position giving two series of data with three line-fluxes per spectrum, see Figures 8, 9, 10, and 11; for example, the west position will sample the south-west and the north-west regions of the wind. The two sequences in every panel in the Figures represent these two series. In Figure 8 the decline of the added $\mathrm{R}(1), \mathrm{R}(2)$ and $\mathrm{R}(3)$ intensities for the four slit positions are shown.

Table 1 gives the observed intensities of the CO emission lines from the spectra representing the closest points to the star, i.e. $(2 \pm 0.5)^{\prime \prime}$ away from the star, as well as the mean intensity ratios, $\mathrm{R}(1) / \mathrm{R}(3)$ and $\mathrm{R}(2) / \mathrm{R}(3)$, for positions from $2.0^{\prime \prime}$ to $3.4^{\prime \prime}$. Since the $\mathrm{S} / \mathrm{N}$ ratio at the observed spatial resolution is too low, the data will unfortunately not permit a useful plot of the intensity ratios as a function of distance; The scatter is too large. The best-fit slopes of the intensity as a function of angular distance from the star, $\operatorname{dlog} \mathrm{I} / \mathrm{d} \log \beta$, for the $\mathrm{R}(2)$ emission line are also given in the Table. The uncertainties quoted are pure measuring uncertainties, i.e. they do not include possible systematic uncertainties. We obtained the best signal for our west position. The intensity decreases by a factor of about 40 from $2^{\prime \prime}$ to $7^{\prime \prime}$. A mean slope of the west, east, and north posi- tions is $\mathrm{d} \log I / \mathrm{d} \log \beta=-2.8 \pm 0.3$. Here we omit the south measurement in order to lower the uncertainty. Naturally, the signal-to-noise ratios decrease rapidly outwards. The uncertainty quoted is the measuring uncertainty.

The $R(1)$ emission line is situated close to the left (red) edge of the detector. The spectra show a peculiar rise in intensity here which makes the measured values of the $R(1)$ lines subject to a systematic uncertainty. Especially the east measurement of the $\mathrm{R}(1)$ line seems to be stronger than expected when compared with the measurements in the other directions. The $\mathrm{R}(1) / \mathrm{R}(3)$ ratios are also affected by this effect. The east and north spectra in Figure 6 show a spurious decline at lower wavenumbers (at the red end). This is due to a mismatch in intensity between the on- and off-star spectra at the edge of the detector array probably not caused by flat-fielding or other reduction procedures. This spurious effect may, unfortunately, affect all frames.

\section{DISCUSSION}

We find a power-law dependence of the intensity as a function of angular distance on the sky, $\mathrm{I} \propto \beta^{-3}$. Furthermore, in view of various uncertainties, e.g. in the positioning of the slit, we find that the measured emission line intensities in different directions from the star are consistent with a symmetric wind. The wind is at least symmetric to within a factor of two in density. A true west and east position of $1.8^{\prime \prime} \mathrm{W}$ and $2.2^{\prime \prime} \mathrm{E}$ from the star would yield our measured values for a symmetric wind. Corresponding values for the north and south positions would be $1.7^{\prime \prime} \mathrm{N}$ and $2.3^{\prime \prime} \mathrm{S}$. Thus, a small error in the position of $0.2-0.3^{\prime \prime}$ (which is approximately the accuracy of the positioning of the slit) in the measurement from a symmetric wind would yield the 'asymmetric' values we measured.

The relative intensities measured on the two nights can be checked at the cross-over points, cf. for example Figure 8. The intensities differ by approximately $50 \%$, probably reflecting the uncertainties in the slit positions, but also the accuracy of the absolute flux calibration between the nights. The flux calibrations of the observations on the two nights are based on two different onstar measurements, which are subject to different 
uncertainties, as discussed in Section 4.

However, a comparison of the two direction in one slit position may reveal asymmetries in the wind. In Figures 9, 10, and 11, which represent the three lines measured, the increase in intensity at $3-4^{\prime \prime}$ in the 'north-east' data in the 'north'panel is seen in all transitions. This is also the case for the 'south-east' data in the 'south'-panel which all show approximately the same morphology. Thus, this could indicate that there is an east-west asymmetry in the intensity measured, reflecting an asymmetric distribution of $\mathrm{CO}$. This could be due to the additional bipolar outflow detected at larger scales in the aperture synthesis CO maps by Planesas et al. (1990b). Note that their highest resolution is approximately $6^{\prime \prime}$.

We now make a simple analytic model of our observations by assuming a spherically symmetric and homogeneous wind with a constant mass-loss rate and a constant expansion velocity. We also assume the wind to be optically thin in the CO lines along the line-of-sight for rays from regions beyond a certain distance from the star. The adequacy of this latter assumption will be investigated below. Based on Eq. (5) by Gustafsson et al. (1997) the ratio of the wavelength-integrated, line-scattered intensity, $\mathrm{I}_{\mathrm{CO}, \mathrm{i}}\left(\mathrm{erg} \mathrm{s}^{-1} \mathrm{~cm}^{-2} \operatorname{arcseconds}^{-2}\right)$, and the line-scattering flux $\overline{\mathrm{f}}_{\lambda}\left(\mathrm{erg} \mathrm{s}^{-1} \mathrm{~cm}^{-2} \mathrm{~cm}^{-1}\right)$ as seen by the scattering molecules averaged across the line width but measured at the distance $d$, is found to be

$$
\begin{aligned}
\frac{\mathrm{I}_{\mathrm{CO}, \mathrm{i}}(\beta)}{\overline{\mathrm{f}}_{\lambda}}= & \frac{206265}{32} \frac{e^{2} \lambda^{2}}{m_{e} c^{2} m_{H}} f_{u \leftarrow l} \dot{\mathrm{M}} \times \\
& \frac{\mathrm{N}_{\mathrm{i}}(\mathrm{CO}) / \mathrm{N}(\mathrm{CO}) \cdot \epsilon_{\mathrm{CO}}}{\mu v_{e} \mathrm{~d}}\left(\frac{1}{\beta}\right)^{3},
\end{aligned}
$$

where $f_{u \leftarrow l}$ is the absorption oscillator strength of the line, $\epsilon_{\mathrm{CO}}$ is the fractional abundance of $\mathrm{CO}$ molecules, i.e. $[\mathrm{CO}] /[\mathrm{H}]$, and $\mathrm{N}_{i}(\mathrm{CO})$ denotes the number density of $\mathrm{CO}$ molecules in the lower state, $i$, of the transition. Furthermore, $\mu$ is the mean molecular weight, $\mathrm{d}$ is the distance to the star, $v_{\mathrm{e}}$ is the terminal expansion wind velocity and $\beta$ is the angular distance from the star on the sky in seconds of arc. A feature of this general expression is the minus third power dependence of the scattered intensity as a function of the impact parameter on the sky, $\beta$, which agrees within the uncertainties with our observation (cf. Figure 8). Eq. (1) will now be applied to the data for the $\mathrm{R}(2)$ line for which our data have the highest quality.

The oscillator strength $f_{u \leftarrow l}$ of the $\mathrm{R}(2)$ vibrationrotation CO-line at $4.6 \mu \mathrm{m}$ is $6.1 \times 10^{-6}$ (KirbyDocken and Liu 1978 provide oscillator strengths, which for our transitions are consistent with Goorvitch \& Chackerian (1994) and Huré \& Roueff $(1996)^{3}$. See also Table 2). Thus, from Eq. (1) we find that the mass-loss rate as deduced from the emitted intensity from the $\mathrm{R}(2)$ line of $\mathrm{CO}$, and, assuming $\mu \approx 1.2$, is

$$
\begin{aligned}
\dot{\mathrm{M}}= & 4.20 \times 10^{-10} \cdot \frac{v_{e} \mathrm{~d} \beta^{3}}{\mathrm{~N}_{\mathrm{i}}(\mathrm{CO}) / \mathrm{N}(\mathrm{CO}) \cdot \epsilon_{\mathrm{CO}}} \times \\
& \frac{\mathrm{I}_{\mathrm{CO}, \mathrm{i}}(\beta)}{\overline{\mathrm{f}}_{\lambda}}\left(\mathrm{M}_{\odot} \mathrm{yr}^{-1}\right),
\end{aligned}
$$

where $v_{\mathrm{e}}$ is given in $\mathrm{km} \mathrm{s}^{-1}$, $\mathrm{d}$ in parsecs, $\beta$ in seconds of arc, $\mathrm{I}_{\mathrm{CO}, \mathrm{i}}$ in $\mathrm{erg} \mathrm{s}^{-1} \mathrm{~cm}^{-2} \operatorname{arcseconds}^{-2}$, and $\overline{\mathrm{f}}_{\lambda}$ in $\operatorname{erg~s}^{-1} \mathrm{~cm}^{-2} \mu \mathrm{m}^{-1}$. The fractional abundance of ${ }^{12} \mathrm{CO}$ molecules relative to hydrogen, $\epsilon_{\mathrm{CO}}$, is assumed to be constant throughout the envelope and it is assumed that most oxygen is locked-up as $\mathrm{CO}$ molecules. From the literature we find $\mathrm{f}_{\mathrm{CO}}=[\mathrm{CO}] /\left[\mathrm{H}_{2}\right]=5 \times 10^{-4}$ (Knapp et al. 1998), which means that $\epsilon_{\mathrm{CO}}=2.5 \times 10^{-4}$. We now need to estimate the fraction $\mathrm{N}_{\mathrm{i}}(\mathrm{CO}) / \mathrm{N}(\mathrm{CO})$ of $\mathrm{CO}$ molecules that are excited to the $\left(v^{\prime \prime}=\right.$ $0, J^{\prime \prime}=2$ )-level. We assume the population to be controlled by radiation transitions between the $v^{\prime \prime}=0$ and $v^{\prime \prime}=1$ states. This radiation is originally supplied by the stellar photosphere at roughly the stellar effective temperature, $T_{\text {eff }}$, but diluted by a factor of $\left(\mathrm{R}_{*} / r\right)^{2}$ at a distance $r$ from the star. Neglecting all loss or addition of photons in the optically thick spectral lines we find a characteristic temperature of the radiation $T_{r}$ from

$$
B\left(\lambda, T_{r}\right) \approx B\left(\lambda, T_{\mathrm{eff}}\right) \times\left(\mathrm{R}_{*} / r\right)^{2} .
$$

This estimate gives a radiation temperature at a distance of $3^{\prime \prime}$ of about $340 \mathrm{~K}$, suggesting an $\mathrm{N}_{\mathrm{i}=2}(\mathrm{CO}) / \mathrm{N}(\mathrm{CO})$-value of approximately 4\%. This temperature is several times higher than the kinetic temperature of the gas (the level is super-thermally excited). Detailed numerical

\footnotetext{
${ }^{3}$ Note the missing cube in their Eq. (3)
} 
simulations of the radiative transfer in an envelope model of $o$ Ceti by Ryde \& Schöier (2000) verifies the assumption of radiationally controlled populations and indicates population fractions, $\mathrm{N}_{\mathrm{i}=2}(\mathrm{CO}) / \mathrm{N}(\mathrm{CO})$, that range from approximately $3 \%$ to $10 \%$ at distances from $2^{\prime \prime}$ to $7^{\prime \prime}$. Here, we have adopted a value $\mathrm{N}_{\mathrm{i}=2}(\mathrm{CO}) / \mathrm{N}(\mathrm{CO})$ of $5 \%$, noting that a numerical modelling of the circumstellar envelope will be necessary for a more detailed and accurate discussion. On the assumption that the terminal wind velocity $v_{\mathrm{e}}=3 \mathrm{~km} \mathrm{~s}^{-1}$, the mass-loss rate deduced from the measured intensity at $2^{\prime \prime}$ away from the star can be written as

$$
\dot{\mathrm{M}}=0.10 \cdot \mathrm{I}_{\mathrm{CO}}(\beta) / \overline{\mathrm{f}}_{\lambda} \quad\left(\mathrm{M}_{\odot} \mathrm{yr}^{-1}\right) .
$$

The observations at $\beta \approx 2^{\prime \prime}$ of the east and west positions give $\mathrm{I}_{\mathrm{CO}}(\beta) / \overline{\mathrm{f}}_{\lambda}=3.1 \times$ $10^{-6} \mu \mathrm{m}\left({ }^{\prime \prime}\right)^{-2}$ and of the south and north positions give $\mathrm{I}_{\mathrm{CO}}(\beta) / \overline{\mathrm{f}}_{\lambda}=2.8 \times 10^{-6} \mu \mathrm{m}\left({ }^{\prime \prime}\right)^{-2}$, where $\overline{\mathrm{f}}_{\lambda}$ is estimated from the on-star spectrum. The mean mass-loss rate found by this analytic discussion is then $\dot{\mathrm{M}}=3.2 \times 10^{-7} \mathrm{M}_{\odot} \mathrm{yr}^{-1}$ for the east and west positions, and $\dot{\mathrm{M}}=2.8 \times 10^{-7} \mathrm{M}_{\odot} \mathrm{yr}^{-1}$ for the north and south positions. The uncertainty in the derived mass-loss rate (which is independent of the flux calibration since we use a ratio of fluxes in the derivation), are due to uncertainties in the position of the slit, the level population, the measured scattered intensity, and the on-star intensity. Considering these sources of uncertainties we estimate a conservative uncertainty in the mass-loss rate of typically a factor of 4 .

Our derived value of the mass-loss rate is in good agreement with values in the literature. For example, Young (1995) arrive at $(3.6 \pm 1.2) \times$ $10^{-7} \mathrm{M}_{\odot} \mathrm{yr}^{-1}$ from the $\operatorname{CO}(J=3-2)$ line for a distance of $100 \mathrm{pc}$ and a $\epsilon_{\mathrm{CO}}=3 \times 10^{-4}$. The largest uncertainty in the estimates of mass-loss rates for miras has until recently been the distance. The Hipparcos satellite (ESA 1997) has radically improved this situation. Young's mass-loss rate corresponds to $(2.8 \pm 0.9) \times 10^{-7} \mathrm{M}_{\odot} \mathrm{yr}^{-1}$ with our values of $\mathrm{d}$ and $\epsilon_{\mathrm{CO}}$. Other estimates of the mass-loss rate lie between $(1-5) \times 10^{-7} \mathrm{M}_{\odot} \mathrm{yr}^{-1}$, see e.g. Danchi et al. (1994), Loup et al. (1993), Planesas et al. (1990a), and Knapp \& Morris (1985). It should be mentioned again that the radio profiles are difficult to model due to their asymmetry and require an additional component apart from for the standard CSE usually used. There may be a bipolar nebula seen pole-on (cf. the discussion in Knapp \& Morris 1985).

An assumption made in Eq. (1) is that the circumstellar envelope is tangentially optically thin, i.e. along the line-of-sight, at distances from the star at which the measurements are made, i.e. 2$7^{\prime \prime}$. This optical depth is easily found from

$$
\begin{aligned}
\tau_{\text {tang }}(p)= & \frac{e^{2} \lambda^{2}}{m_{e} c^{2} m_{H}} \frac{f_{u \leftarrow l} \dot{\mathrm{M}}}{\delta \lambda} \frac{\epsilon_{\mathrm{CO}}}{4 \mu v_{e}} \frac{N_{i}(C O)}{N(C O)} \times \\
& \int_{-L}^{L} \frac{\mathrm{d} l}{p^{2}+l^{2}},
\end{aligned}
$$

where $p$ is the 'impact parameter' which denotes the closest distance from the star at the location on the sky of the observations and $l$ is the variable of integration (tangential distance along the lineof-sight with the closest point to the star as the zero point). The integration should be calculated over a region of the envelope $(-\mathrm{L}<l<\mathrm{L})$ where the differential Doppler shifts are smaller than the width of the line. Approximating the line profile with a step-function, we find this distance to be

$$
2 L \approx p \frac{\delta \lambda}{\lambda} \frac{c}{v_{\mathrm{e}}} .
$$

An estimate of the tangential optical depth of the wind based on the derived mass-loss rate and the estimated expansion velocity, is obtained by solving the integral (Eq. 5). We find that $\tau_{\text {tang }} \approx 7.6 \times$ $10^{15} / p$, where $p$ should be in centimeters. Inwards of approximately $4^{\prime \prime}$ we find that $\tau_{\operatorname{tang}}(p) \gtrsim 1$, i.e. that the wind is expected to be optically thick along a line-of-sight. Although the assumption that the optical depth is smaller than 1 is not fulfilled in the inner part of the observed region $\left(\beta \approx 2-7^{\prime \prime}\right)$, the CO emission declines smoothly as $\beta^{-3}$ all the way in, indicating that the assumption of the wind being optically thin could nevertheless be correct. The dependence on angular distance of the scattered intensity is not expected to show the same behaviour in an optically thick and an optically thin wind. For the KI scattering in the wind of R Scl (Gustafsson et al. 1997) the same situation is found. The $\beta^{-3}$-dependence of the intensity could be preserved throughout the observed parts of the wind if the circumstellar gas were inhomogeneous, which probably is a reasonable assumption, (cf. the discussion in Gustafsson 
et al. 1997). This fact could lead to a lowering of the optical depth if the structures ('clumps') of the wind are of a suitable characteristic size and have a low filling factor in the line-of-sight. Note, that the measured decline of the intensity could also be achieved if the lines are optically thick and the mass-loss rate is not uniform but vary with time. However, this seems to require a fine-tuning of the mass-loss rate with time which does not seem realistic. A numerical simulation of the Mira wind (Ryde \& Schöier 2000) will discuss the optical depths in more detail.

In applying Eq. (2) it was assumed that the line radiation at distance $r$ from the center of the star is represented satisfactorily by the measured on-star flux $\overline{\mathrm{f}}_{\lambda}$, corrected by the distance factor $(d / r)^{2}$. In practise, the observed on-star spectrum will also contain strong contributions from stellar light scattered in the inner envelope in non-radial directions, provided that the gas is not devoid of scatterers there. The velocity divergence then brings the photon out of the core of the line profile at points along the new direction and the chances for it to be re-scattered are minimal. Therefore, a considerable fraction of the photons are lost 'sideways' from the envelope due to scattering already in its inner regions, see also the discussion in Ryde \& Schöier (2000).

\section{CONCLUSIONS}

The successful detection of scattered photospheric light in the circumstellar CO vibrationrotation lines of Mira has made it possible to explore the structure of its intermediate circumstellar envelope (at distances from the star of approximately 100 - 1000 stellar radii).

The variation of the emission with angular distance from the star $\left(2^{\prime \prime}<\beta<7^{\prime \prime}\right)$ is found to roughly follow a $\beta^{-3}$ behavior, $\beta$ being the angular distance on the sky. Our data indicate that the mass-loss rate, when averaged over about 100 years, was constant over a period of about 1200 years, assuming that the wind is optically thin along the line-of-sight for the measured angular distances.

Our observations provide absolute fluxes scattered in the circumstellar CO R(1), R(2), and R(3) lines, and provide corresponding line ratios. Based on a simple analytic model, the mass-loss rate is estimated to be approximately $3 \times 10^{-7} \mathrm{M}_{\odot} \mathrm{yr}^{-1}$ which, in view of the uncertainties, is compatible with earlier estimates in the literature. A numerical modelling of the wind will be necessary for a more detailed and accurate discussion of the circumstellar envelope. Such a modelling will be presented in a forthcoming paper (Ryde \& Schöier 2000).

We have also found that the envelope is approximately spherically-symmetric to within a factor of two in density. Note, however, the asymmetries found by, for example, Planesas et al. (1990b). We have found indications that a similar asymmetry is also present in the $\mathrm{CO}$ vibration-rotation emission intensity at a distance of approximately $3^{\prime \prime}$ from the star in the east direction as compared with the west direction. It is interesting to note that the companion of Mira is located eastward from the star at a position angle of $(108.3 \pm 0.1)^{\circ}$ (Karovska et al. 1997).

The referee is thanked for very valuable comments. We should also like to thank J. Valenti for assistance during the observations, and H. Olofsson for stimulating discussions. We are grateful to J. Barnes, L. Borgonovo, K. Ryde, and N. Piskunov for generous assistance. We also owe a dept of thanks to S. Höfner and M. Asplund for valuable comments on the manuscript. We acknowledge financial support from the Swedish National Space Board and the Royal Swedish Academy of Sciences.

\section{REFERENCES}

Bernat, A. P. and Lambert, D. L., 1975, ApJ, 201, L153

Bernat, A. P. and Lambert, D. L., 1976, ApJ 210, 395

Bowen, G. H., 1988, ApJ 329, 299

Clegg, P., Ade, P., Armand, C., et al., 1996, A\&A 315, L38

Crosas, M., Menten, K. M., Young, K., and Phillips, T. G., 1997, Ap\&SS 251, 189

Danchi, W. C., Bester, M., Degiacomi, C. G., Greenhill, L. J., and Townes, C. H., 1994, AJ 107, 1469 
Deutsch, A. J., 1956, ApJ 123, 210

Dorfi, E. A. and Höfner, S., 1996, A\&A 313, 605

Dyck, H. M., Beckwith, S., and Zuckerman, B., 1983, ApJ Letters 271, L79

ESA, 1997, ESA, The Hipparcos and Tycho Catalogues, ESA SP-1200

Gezari, D. Y., Schmitz, M., and Mead, J. M., 1984, Catalog of infrared observations including: Bibliography of infrared astronomy and index of infrared source positions

Goorvitch, D., 1994, ApJS 95, 535

Goorvitch, D. and Chackerian, C., 1994, ApJS 91, 483

de Graauw, T., van den Ancker, M., Bauer, O. H., et al., 2000, in The ISO Handbook; SWSthe Short Wavelength Spectrometer, SAI/2000008/Dc. Version 1.0, p. 96

Guilain, C. and Mauron, N., 1996, A\&A 314, 585

Gustafsson, B., Bell, R. A., Eriksson, K., and Nordlund, A., 1975, A\&A 42, 407

Gustafsson, B., Eriksson, K., Kiselman, D., Olander, N., and Olofsson, H., 1997, A\&A 318, 535

Habing, H. J., 1996, A\&AR 7, 97

Haniff, C. A., Scholz, M., and Tuthill, P. G., 1995, MNRAS 276, 640

Hinkle, K. H., Cuberly, R. W., Gaughan, N. A., et al., 1998, SPIE 3354, 810

Hinkle, K. H., Hall, D. N. B., Ridgway, S. T. 1982, ApJ 252, 697

Hinkle, K. H., Scharlach, W. W. G., Hall, D. N. B. 1984, ApJS 56, 1

Höfner, S. and Dorfi, E. A., 1997, A\&A 319, 648

Höfner, S., Jørgensen, U. G., Loidl, R., and Aringer, B., 1998, A\&A 340, 497

Huré, J. M. and Roueff, E., 1996, A\&AS 117, 561

Joyce, R. R., 1992, in S. Howell (ed.), ASP Conf. Ser. 23: Astronomical CCD Observing and Reduction Techniques, p. 258
Karovska, M., Hack, W., Raymond, J., and Guinan, E., 1997, ApJ 482, L175

Karovska, M., Nisenson, P., and Beletic, J., 1993, ApJ 402, 311

Kessler, M. F., Steinz, J. A., Anderegg, M. E., et al., 1996, A\&A 315, L27

Kirby-Docken, K. and Liu, B., 1978, ApJS 36, 359

Knapp, G. R., Morris, M., 1985, ApJ 292, 640

Knapp, G. R., Young, K., Lee, E., and Jorissen, A., 1998, ApJS 117, 209

Léna, P., 1988, in Observational Astrophysics, Springer Verlag, p. 256

Loup, C., Forveille, T., Omont, A., and Paul, J. F., 1993, A\&AS 99, 291

Mahler, T. A., Wasatonic, R., and Guinan, E. F., 1997, Informational Bulletin on Variable Stars 4500, 1

Olofsson, H., Bergman, P., Eriksson, K., and Gustafsson, B., 1996, A\&A 311, 587

Partrige, H. and Schwencke, D., 1997, J. Chem. Phys. 106, 4618

Perrin, G., Coude Du Foresto, V., Ridgway, S. T., et al., 1998, A\&A 331, 619

Planesas, P., Bachiller, R., Martin-Pintado, J., and Bujarrabal, V., 1990a, ApJ 351, 263

Planesas, P., Kenney, J .D. P., and Bachiller, R., 1990b, ApJ 364, L9

Plez, B. et al., 2000, in preparation

Plez, B. and Lambert, D. L., 1994, ApJ 425, L101

Ryde, N., Gustafsson, B., Hinkle, K. H., Eriksson, K., Lambert, D. L., and Olofsson, H., 1999, A\&A 347, L35

Ryde, N. \& Schöier, F.L., 2000, submitted

Sahai, R. and Wannier, P. G., 1985, ApJ 299, 424

Van Leeuwen, F., Feast, M. W., Whitelock, P. A., and Yudin, B., 1997, MNRAS 287, 955

Woitke, P., 1998, in Cyclical Variability in Stellar Winds, Springer Verlag, p. 278 
Young, K., 1995, ApJ 445, 872

This 2-column preprint was prepared with the AAS IATEX macros v5.0. 
Fig. 1.- The slit positions for our observations of Mira's envelope. While the spectrograph slit was $30^{\prime \prime}$ long, the slit indicated in the Figure is 14 " long to indicate the regions in which we detected resonance-scattered $\mathrm{CO}$ emission. The underlying intensity map is taken from Planesas et al. (1990b). It shows the $\mathrm{CO}(J=2-1)$ line emission obtained with the IRAM $30 \mathrm{~m}$ telescope, in this case for $47.7 \mathrm{~km} \mathrm{~s}^{-1}$, which is close to the stellar systemic velocity. The difference between two contour lines is $2 \mathrm{~K} \mathrm{~km} \mathrm{~s}^{-1}$. The IRAM beam is 13 " in diameter.

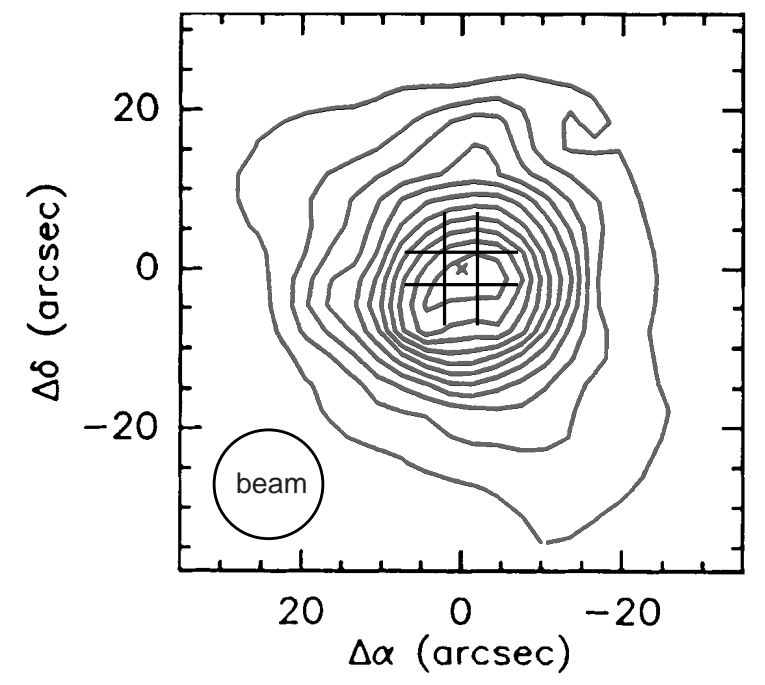


Fig. 2.-A low-resolution ISO spectrum $(\mathrm{R} \approx 100)$ is shown in full line. The dashed line indicates the transmission profile of the Johnson M-band used in the absolute flux calibration of the observations. The width of the tall box denotes the observed spectral range of the Phoenix spectrometer.

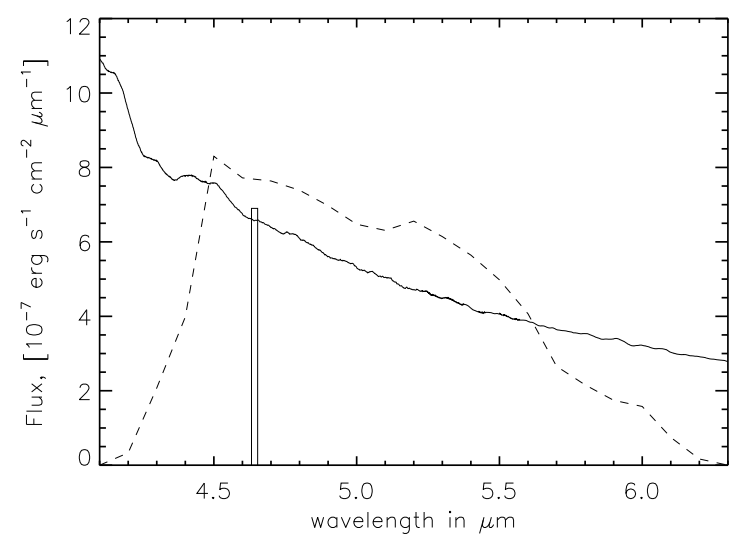


Fig. 3.- The observed on-star spectrum of Mira is shown as a full line. The superimposed dashed line represents the synthetic spectrum generated from a OS-MARCS model atmosphere for the stellar parameters $\mathrm{T}_{\mathrm{eff}}=2400 \mathrm{~K}, \log g=-1.0[\mathrm{cgs}]$, and solar metallicity and assuming a stellar radius of $3.8 \cdot 10^{13} \mathrm{~cm}$ and a distance of $128 \mathrm{pc}$. The model fluxes are multiplied by a factor of 1.4 in order to match the observed spectrum. The agreement is astonishingly good, suggesting that the absolute flux calibration is reliable. The dominating spectral features are due to photospheric water vapor and CO. The telluric water and CO lines are marked $(\oplus)$. Also, the absorption lines due to the photospheric CO 1-0 R(1), R(2), and $\mathrm{R}(3)$ transitions are indicated in the Figure. Circumstellar absorption and emission is notable in the cores of the CO lines, especially R(3).

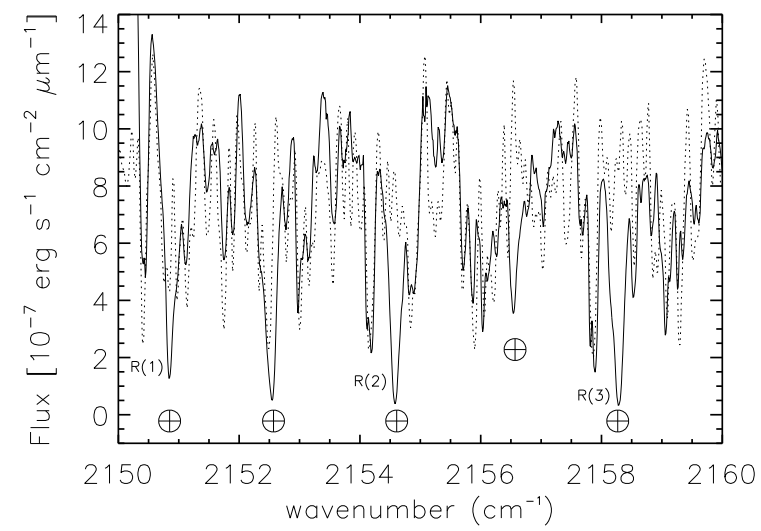


Fig. 4.- The observed scattered spectrum from an off-star observation including the $\mathrm{R}(2)$ line is presented as a dotted line. The solid line indicates a scaled on-star spectrum. From the Figure we can see that the small emission peak in the photospheric CO $\mathrm{R}(2)$ absorption line is located at the same wavenumber as the off-star scattered spectrum. This is the result of some residual circumstellar emission in the on-star spectrum. Circumstellar absorption along the line-of-sight through the circumstellar shell is present to the right (blue shifted) from the emission. The on-star emission and absorption components should nearly cancel in the measured scattered intensities, which are the differences of our off- and on-star spectra.

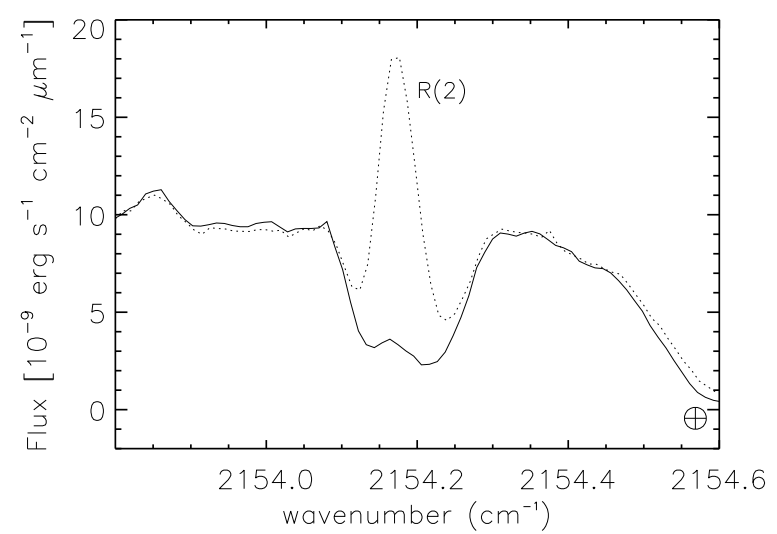


Fig. 5.- The spectra of $o$ Ceti (Mira) with the abscissa showing the wavenumber scale of the observer. The off-star spectrum is shown by a dotted line and the appropriately scaled on-star spectrum is shown by a solid line. The three circumstellar $\mathrm{CO}$ vibration-rotation emission lines, which are marked $\mathrm{R}(1), \mathrm{R}(2)$, and $\mathrm{R}(3)$, 'fill in' the corresponding on-star lines which are in absorption. To the right (blue) of these lines the telluric $\mathrm{CO}$ vibration-rotation $\mathrm{R}(1), \mathrm{R}(2)$, and $\mathrm{R}(3)$ lines are seen. These telluric CO lines and the two major telluric water vapor lines at 2152.5 and $2156.5 \mathrm{~cm}^{-1}$ are denoted with $\oplus$-symbols. The stellar CO lines are shifted out of the telluric ones.

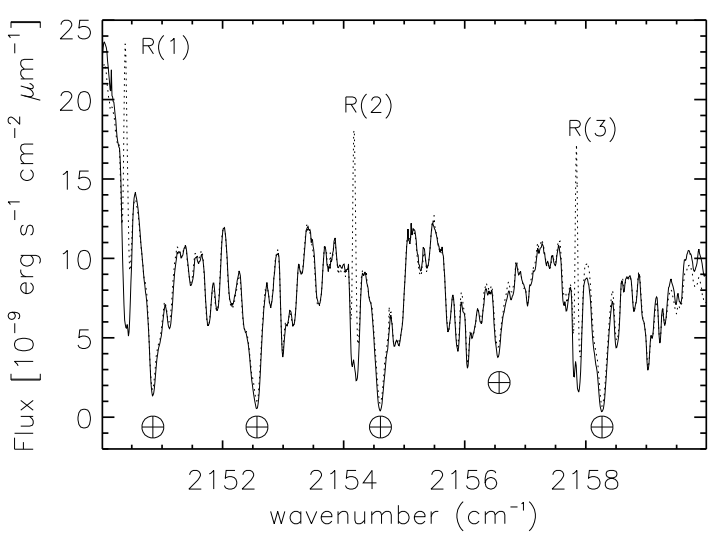


Fig. 6.- The resulting circumstellar $\mathrm{CO}$ vibration-rotation flux from the wind of $o$ Ceti. The intensity is integrated over the full slit area. A scaled on-star spectrum is subtracted from the off-star spectrum. In the four panels the spectra from the observations with the slit lying tangentially $2^{\prime \prime} \mathrm{W}, \mathrm{E}, \mathrm{N}$, and $\mathrm{S}$, respectively, of the star are shown. Note the different ordinate scales.

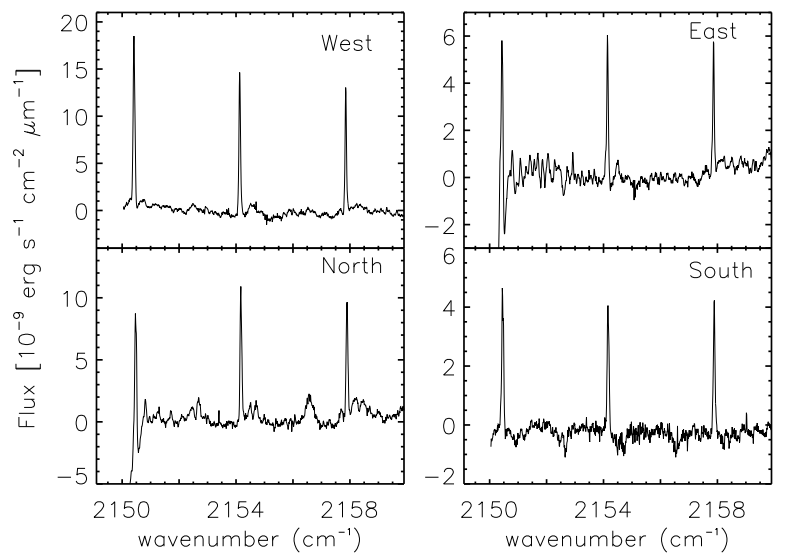


Fig. 7.- A set of emission spectra from the west position is shown to indicate the signal-tonoise ratio at different distances from the star. The spectra are shifted vertically with respect to each other. The top spectrum is measured closest to the star, i.e. at a distance of $2^{\prime \prime}$. The subsequent spectra are measured at $2.2^{\prime \prime}$, $2.8^{\prime \prime}, 3.6^{\prime \prime}$, and $4.5^{\prime \prime}$ away from the star, respectively. The intensity scale is given in units of $10^{-14} \mathrm{erg} \mathrm{s}^{-1} \mathrm{~cm}^{-2} \operatorname{arcsec}^{-2} \mu \mathrm{m}^{-1}$.

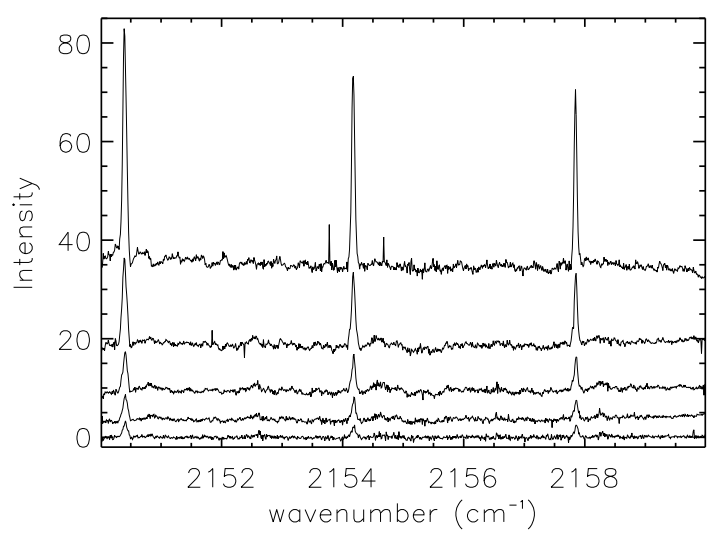


Fig. 8.- The decline of the intensity of the circumstellar emission as a function of angular distance $(\beta)$ from the star. The line is a pure power law $\left(\mathrm{I} \propto \beta^{-3}\right)$. The intensities of the three vibration-rotation lines $[R(1), R(2)$, and $R(3)]$ are added in order to increase the signal-to-noise ratio. The two different symbols represent the two different directions along the long-slit, as indicated in the different panels.

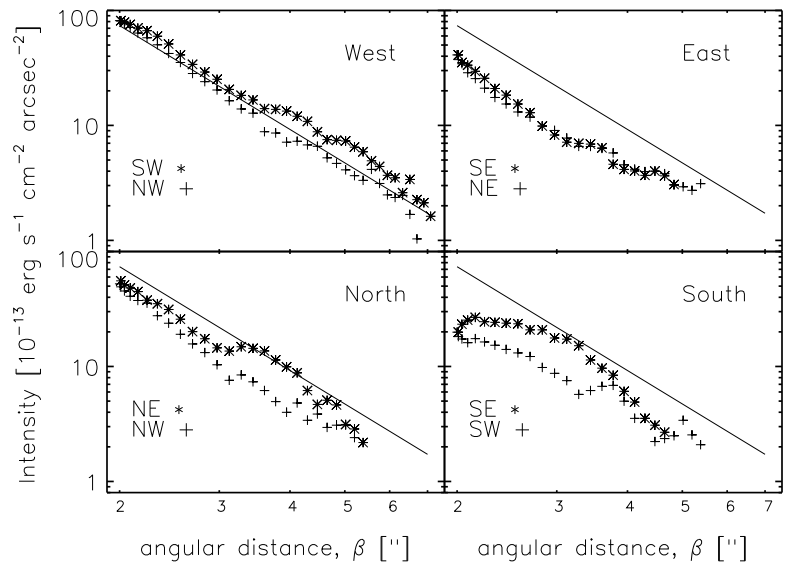


Fig. 9.- The decline of the intensity of the circumstellar emission as a function of angular distance $(\beta)$ from the star, as in Figure 8. Here the scattered light of the $\mathrm{CO} R(1)$ vibration-rotation line is shown.

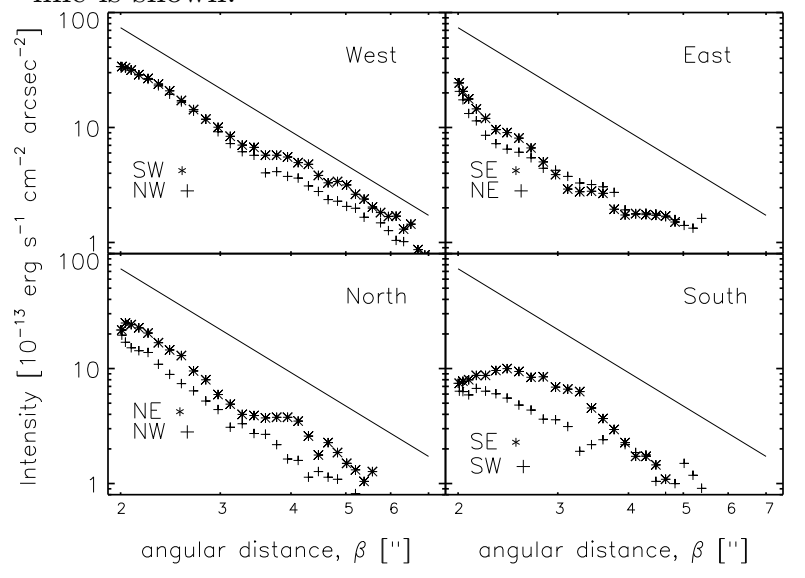


Fig. 10. - The decline of the intensity of the circumstellar emission as a function of angular distance $(\beta)$ from the star, as in Figure 8. Here the scattered light of the $\mathrm{CO} R(2)$ vibration-rotation line is shown.

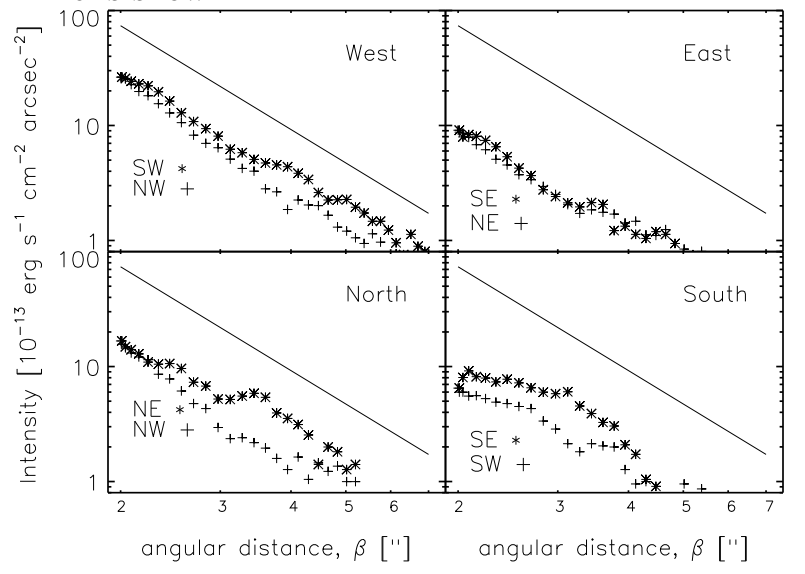


Fig. 11. - The decline of the intensity of the circumstellar emission as a function of angular distance $(\beta)$ from the star, as in Figure 8. Here the scattered light of the $\mathrm{CO} R(3)$ vibration-rotation line is shown.

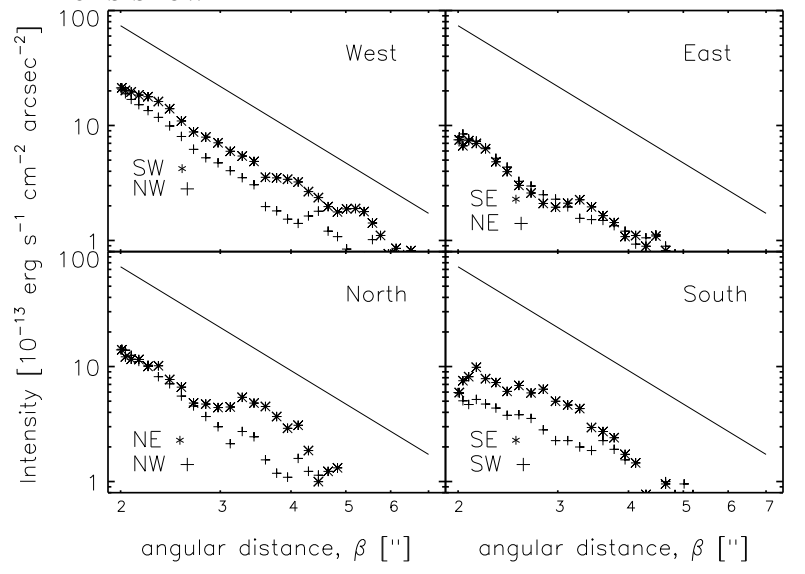


TABLE 1

Measured intensities of the CO Emission lines, meAn INTEnsity RATios AND BEST-Fit SLOPES

\begin{tabular}{|c|c|c|c|c|}
\hline & West & East & North & South \\
\hline \multicolumn{5}{|c|}{ Intensities $\left[\mathrm{erg} \mathrm{s}^{-1} \mathrm{~cm}^{-2} \operatorname{arcseconds}^{-2}\right]$ : } \\
\hline $\mathrm{I}_{\mathrm{R}(1)}$ & $3.3 \times 10^{-12}$ & $2.0 \times 10^{-12}$ & $2.3 \times 10^{-12}$ & $0.9 \times 10^{-12}$ \\
\hline $\mathrm{I}_{\mathrm{R}(2)}$ & $2.6 \times 10^{-12}$ & $0.9 \times 10^{-12}$ & $1.5 \times 10^{-12}$ & $0.8 \times 10^{-12}$ \\
\hline $\mathrm{I}_{\mathrm{R}(3)}$ & $2.0 \times 10^{-12}$ & $0.8 \times 10^{-12}$ & $1.3 \times 10^{-12}$ & $0.8 \times 10^{-12}$ \\
\hline
\end{tabular}

Line ratios:

$\begin{array}{lllll}\mathrm{R}(1) / \mathrm{R}(3) & 1.7 \pm 0.2 & 2.1 \pm 0.6 & 1.7 \pm 0.3 & 1.2 \pm 0.2 \\ \mathrm{R}(2) / \mathrm{R}(3) & 1.3 \pm 0.1 & 1.2 \pm 0.2 & 1.2 \pm 0.2 & 1.1 \pm 0.1\end{array}$

Slopes of $\mathrm{R}(2), \mathrm{d} \log I_{\mathrm{R}(2)} / \mathrm{d} \log \beta$ :

$\begin{array}{llll}-3.3(\mathrm{NW}) & -2.5(\mathrm{NE}) & -3.0(\mathrm{NW}) & -2.3(\mathrm{SW}) \\ -2.8(\mathrm{SW}) & -2.5(\mathrm{SE}) & -2.7(\mathrm{NE}) & -3.2(\mathrm{SE})\end{array}$


TABLE 2

Transition PRobabilities FOR SOME CO LINES

\begin{tabular}{cccc}
\hline \hline Line $\left(J^{\prime \prime}-J^{\prime}\right)$ & wavenumber $\left[\mathrm{cm}^{-1}\right]$ & $\mathrm{f}_{J^{\prime \prime} \rightarrow J^{\prime}}$ & $\mathrm{A}_{J^{\prime \prime} \leftarrow J^{\prime}}$ \\
\hline $\mathrm{R}(1)(1-2)$ & 2150.856 & $6.791 \times 10^{-6}$ & 12.57 \\
$\mathrm{R}(2)(2-3)$ & 2154.596 & $6.127 \times 10^{-6}$ & 13.55 \\
$\mathrm{R}(3)(3-4)$ & 2158.300 & $5.850 \times 10^{-6}$ & 14.14 \\
\hline
\end{tabular}

